\title{
Density-dependent effects on Turing patterns and steady state bifurcation in a Beddington-DeAngelis-type predator-prey model
}

\author{
Hongwu $\mathrm{Xu}^{1}$ and Shengmao Fu${ }^{2 *}$ (1)
}

"Correspondence:

fusm@nwnu.edu.cn

${ }^{2}$ School of Mathematics and

Statistics, Northwest Normal

University, Lanzhou, P.R. China

Full list of author information is

available at the end of the article

\section{空 Springer}

\begin{abstract}
In this paper, Turing patterns and steady state bifurcation of a diffusive Beddington-DeAngelis-type predator-prey model with density-dependent death rate for the predator are considered. We first investigate the stability and Turing instability of the unique positive equilibrium point for the model. Then the existence/nonexistence, the local/global structure of nonconstant positive steady state solutions, and the direction of the local bifurcation are established. Our results demonstrate that a Turing instability is induced by the density-dependent death rate under appropriate conditions, and both the general stationary pattern and Turing pattern can be observed as a result of diffusion. Moreover, some specific examples are presented to illustrate our analytical results.
\end{abstract}

MSC: 92D25; 35K57; 35B35

Keywords: Predator-prey model; Density-dependent; Turing instability; Bifurcation; Steady state

\section{Introduction}

Understanding the dynamical relationship between predator and prey is a central research subject in ecology, and one significant component of the predator-prey relationship is the predator's rate of feeding upon prey, i.e., the so-called functional response. Functional response is a double rate: It is the average number of prey killed per individual predator per unit of time. In general, the functional response can be classified into two types: Prey-dependent and predator-dependent. Prey dependence means that the functional response is only a function of the prey's density, while predator dependence means that the functional response is a function of both the prey's and the predator's densities. For the functional response functions, there are many types, such as the Holling family which are predominant in the literature [1].

Since 1959, the Holling II-type prey-dependent functional response has served as the basis for a very large literature on predator-prey theory [2]. However, the prey-dependent functional responses fail to describe the interference among predators, and have been facing challenges from the biology and physiology communities [3, 4]. Some biologists have

(c) The Author(s) 2019. This article is distributed under the terms of the Creative Commons Attribution 4.0 International License (http://creativecommons.org/licenses/by/4.0/), which permits unrestricted use, distribution, and reproduction in any medium, provided you give appropriate credit to the original author(s) and the source, provide a link to the Creative Commons license, and indicate if changes were made. 
argued that in many situations, especially when predators have to search for food (and therefore, have to share or compete for food), the functional response in a predator-prey model should be predator-dependent. There is much significant evidence to suggest that predator dependence in the functional response occurs quite frequently in laboratory and natural systems $[5,6]$. Given that large numbers of experiments and observations suggest that predators do indeed interfere with one another's activities so as to result in competition effects and that prey alters its behavior under increased predator-threat, the models with predator-dependent functional response stand as reasonable alternatives to the models with prey-dependent functional response [2]. Starting from this argument and the traditional prey-dependent model, to describe mutual interference among predators, Beddington [7] and DeAngelis [8] proposed that an individual from a population of more than two predators not only allocates time in searching for and processing their prey but also takes time in encountering other predators. This result in the so-called BeddingtonDeAngelis functional response $p(u, v)=\frac{m u}{a+u+b v}$. The Beddington-DeAngelis functional response is similar to the well-known Holling type II functional response, but it has an extra term $b v$ in the denominator modelling mutual interference among predators, and it also has some similar qualitative features as the ratio-dependent form but avoids the singular behaviors of ratio-dependent models at low densities which have been the source of controversy.

We know the classical Beddington-DeAngelis-type predator-prey system which has received considerable attention [9-17] and takes the form

$$
\left\{\begin{array}{l}
\frac{d u}{d t}=u(1-u)-\frac{m u v}{a+u+b v}, \\
\frac{d v}{d t}=s v\left(-q+\frac{m u}{a+u+b v}\right) .
\end{array}\right.
$$

A salient statistical evidence from nineteen predator-prey systems prove that Beddington-DeAngelis functional response provides better description of predator feeding over a range of predator-prey abundances [2]. In some cases, it performs even better than other functional responses. The most crucial finding of Skalski and Gilliam [2] was that predator dependence in the functional response is a nearly ubiquitous property of the published data sets. Cantrell and Cosner [10] have partially analyzed the dynamics of the system (1). Hwang [13] has solved the problem for the uniqueness of a limit cycle of the system (1). A detailed mathematical analysis of the dynamics for (1) with unlimited carrying capacity for prey population was presented in [14]. Further, Kartina [15] found that predator dependence is important at not only very high predator densities on per capita predation rate but also at low predator densities. In ecology, we should consider the predator density dependence, and we need to take into account realistic levels of predator dependence.

In this paper, we consider the following density-dependent Beddington-DeAngelis-type predator-prey model:

$$
\left\{\begin{array}{l}
\frac{d u}{d t}=u(1-u)-\frac{m u v}{a+u+b v}, \\
\frac{d v}{d t}=s v\left(-q-\delta v+\frac{m u}{a+u+b v}\right),
\end{array}\right.
$$

where $u$ and $v$ represent prey and predator densities, respectively. $q$ is the death rate of the predator, $s$ is the feed concentration and $\delta$ is the density-dependent death rate. Biologically speaking, the positive density-dependent death rate $\delta$ has depressing effect on the growth rate of the predator, i.e., causes the reduction in predator growth rate [16]. 
In [17], the authors studied the dynamics of (2). They proved the permanence, locally and globally asymptotic stability of the positive equilibrium for the model (2) by using stability theory of differential equations and Lyapunov functions. For the permanence, they showed that the density dependence for predator gives some negative effect, compared to the models without the density dependence. In addition, the authors compared results for the model with Beddington-DeAngelis functional response on permanence, locally and globally asymptotic stability to the system with Lotka-Volterra interaction or Holling type II functional response or ratio-dependent functional response.

When the densities of the prey and predator are spatially inhomogeneous in a bounded domain, and the prey and predator move randomly-described as Brownian random motion [18-20], we need consider the following reaction-diffusion model:

$$
\begin{cases}u_{t}-d_{1} \Delta u=u(1-u)-\frac{m u v}{a+u+b v}, & x \in \Omega, t>0, \\ v_{t}-d_{2} \Delta v=s v\left(-q-\delta v+\frac{m u}{a+u+b v}\right), & x \in \Omega, t>0, \\ \frac{\partial u}{\partial v}=\frac{\partial v}{\partial v}=0, & x \in \partial \Omega, t>0, \\ u(x, 0)=u_{0}(x) \geq(\not \equiv) 0, \quad v(x, 0)=v_{0}(x) \geq(\not) 0, & x \in \Omega,\end{cases}
$$

where $\Omega$ is a bounded domain with smooth boundary $\partial \Omega$ and $\nu$ is the outward unit normal vector of the boundary $\partial \Omega$. The positive constants $d_{1}$ and $d_{2}$ are the diffusion coefficients of $u(x, t)$ and $v(x, t)$, respectively. $\triangle$ is the Laplacian operator which describes the random moving.

In the case $b=0$, Huang et al. in [21] derived the conditions for the existence of nonconstant steady states of the model (3) with $\delta>0$. At the same time, they proved that the same system without the density-dependent death rate for the predators does not admit pattern formations. Hence, in the case $b>0$, a natural question is raised: Is the densitydependent death rate $\delta$ also a decisive factor inducing Turing instability in the model (3)? We will answer this problem in this paper.

To study the stationary patterns, we need consider the steady state problem associated with (3)

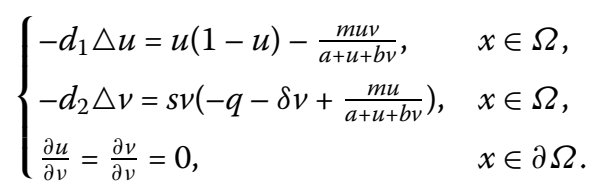

The rest of this paper is organized as follows: In Sect. 2, the stability and Turing instability of the positive equilibrium point $\left(u^{*}, v^{*}\right)$ in (3) are discussed. In Sect. 3, we investigate the nonexistence/existence of nonconstant positive steady states. In Sect. 4, the local and global structure of nonconstant positive steady state are established, and the direction of the local bifurcation is given.

\section{Stability and Turing instability of positive equilibrium point}

In this section, we mainly discuss the stability and Turing instability of the positive equilibrium point of (3). For convenience, we denote

$$
f(u, v)=u(1-u)-\frac{m u v}{a+u+b v}, \quad g(u, v)=s v\left(-q-\delta v+\frac{m u}{a+u+b v}\right) .
$$


Obviously, the model (2) has a trivial equilibrium point $E_{0}=(0,0)$, a semitrivial equilibrium point $E_{1}=(1,0)$ and at least one positive equilibrium point $E^{*}=\left(u^{*}, v^{*}\right)$ if

$$
m>(a+1) q,
$$

where

$$
u^{*}=\frac{b \delta v^{* 2}+(a \delta+b q) v^{*}+a q}{m-q-\delta v^{*}}
$$

and $v^{*}$ is the positive roots of polynomial equation

$$
\begin{aligned}
& \delta\left(b^{2}+\delta\right) v^{3}+\left[(2 a b+b-2 m+2 q) \delta+b^{2} q\right] v^{2} \\
& \quad+\left[\left(a^{2}+a\right) \delta+q^{2}+(2 a b+b-2 m) q+m(m-b)\right] v+a[(a+1) q-m]=0 .
\end{aligned}
$$

To illustrate the uniqueness of the positive equilibrium point, we first give the following lemma.

Lemma 1 (Shengjins discriminant [22]) For the equation $x^{3}+B x^{2}+C x+D=0$, where $B, C, D \in \mathbf{R}$, denote $\mathbb{A}=B^{2}-3 C, \mathbb{B}=B C-9 D, \mathbb{C}=C^{2}-3 B D$ and $\Delta=\mathbb{B}^{2}-4 \mathbb{A}$.

(i) The equation has three real roots if and only if $\Delta \leq 0$.

(ii) The equation has one real root and a pair of conjugate complex roots if and only if $\Delta>0$.

For Eq. (5), corresponding to Lemma 1, let

$$
\begin{aligned}
& B:=\left[(2 a b+b-2 m+2 q) \delta+b^{2} q\right] /\left[\delta\left(b^{2}+\delta\right)\right], \\
& D:=a[(a+1) q-m] /\left[\delta\left(b^{2}+\delta\right)\right], \\
& C:=\left[\left(a^{2}+a\right) \delta+q^{2}+(2 a b+b-2 m) q+m(m-b)\right] /\left[\delta\left(b^{2}+\delta\right)\right],
\end{aligned}
$$

and

$$
\mathbb{A}:=B^{2}-3 C, \quad \mathbb{B}:=B C-9 D, \quad \mathbb{C}:=C^{2}-3 B D, \quad \Delta:=\mathbb{B}^{2}-4 \mathbb{A} \mathbb{C} .
$$

Then we can obtained the following conclusion.

Theorem 2.1 Assume that

$$
\left(\mathbf{H}_{1}\right) \quad m>(a+1) q, \quad \Delta>0
$$

hold, then Eq. (5) has a unique positive root, and (2) has a unique positive equilibrium point $E^{*}=\left(u^{*}, v^{*}\right)$.

Now we discuss the stability and instability of $E^{*}$ for the ODE model (2) and PDE model (3), respectively. By simple calculation, we can see that the Jacobian matrix of (2) evaluated at $E^{*}$ is given by

$$
J\left(E^{*}\right)=\left(\begin{array}{ll}
a_{11} & a_{12} \\
a_{21} & a_{22}
\end{array}\right),
$$


where

$$
\begin{array}{ll}
a_{11}=\frac{m u^{*} v^{*}}{\left(b v^{*}+a+u^{*}\right)^{2}}-u^{*}, & a_{12}=-\frac{m u^{*}\left(a+u^{*}\right)}{\left(b v^{*}+a+u^{*}\right)^{2}}<0, \\
a_{21}=\frac{m\left(b v^{*}+a\right)}{\left(b v^{*}+a+u^{*}\right)^{2}}>0, & a_{22}=-s v^{*}\left(\delta+\frac{m b u^{*}}{\left(b v^{*}+a+u^{*}\right)^{2}}\right)<0 .
\end{array}
$$

The characteristic equation of $J\left(E^{*}\right)$ is

$$
\eta^{2}-Q \eta+P=0
$$

where

$$
Q=a_{11}+a_{22}, \quad P=a_{11} a_{22}-a_{12} a_{21} .
$$

Obviously that $E^{*}$ is locally asymptotically stable if $Q<0$ and $P>0$. Thus, we can obtain the following theorem.

Theorem 2.2 Assume $\left(\mathbf{H}_{1}\right)$ hold. For the model (2), the following statements are true.

(i) If $m u^{*} v^{*}<\left(u^{*}+\delta s v^{*}\right)\left(b v^{*}+a+u^{*}\right)^{2}+b s m u^{*} v^{*}$ and

$$
\begin{aligned}
&\left(\mathbf{H}_{21}\right) \quad s v^{*} {\left[m^{2} b u^{*} v^{*}+m\left(\delta v^{*}-b u^{*}\right)\left(b v^{*}+a+u^{*}\right)^{2}\right] } \\
&<m^{2}\left(a+u^{*}\right)\left(a+b v^{*}\right)+\delta s v^{*}\left(b v^{*}+a+u^{*}\right)^{4}
\end{aligned}
$$

then equilibrium point $E^{*}$ is locally asymptotically stable.

(ii) If $m u^{*} v^{*}>\left(u^{*}+\delta s v^{*}\right)\left(b v^{*}+a+u^{*}\right)^{2}+b s m u^{*} v^{*}$ or

$$
\begin{aligned}
&\left(\mathbf{H}_{\mathbf{2 2}}\right) s v^{*} {\left[m^{2} b u^{*} v^{*}+m\left(\delta v^{*}-b u^{*}\right)\left(b v^{*}+a+u^{*}\right)^{2}\right] } \\
&>m^{2}\left(a+u^{*}\right)\left(a+b v^{*}\right)+\delta s v^{*}\left(b v^{*}+a+u^{*}\right)^{4}
\end{aligned}
$$

then equilibrium point $E^{*}$ is unstable.

To consider Turing instability of $E^{*}$ for PDE model (3), we denote $0=\lambda_{0}<\lambda_{1}<\cdots$, the sequence of eigenvalues for the problem

$$
-\triangle \phi=\lambda \phi, \quad x \in \Omega, \quad \frac{\partial \phi}{\partial v}=0, \quad x \in \partial \Omega,
$$

and $\lambda_{i}(i \geq 1)$ has multiplicity $m_{i} \geq 1$, whose corresponding normalized eigenfunctions are given by $\phi_{i j}$, where $j=1,2, \ldots, m_{i}$. This set of eigenfunctions form an orthogonal basis in $L^{2}(\Omega)$.

If $a_{11}>0$ and

$$
d_{1} \lambda_{1}<a_{11}
$$

then we define $i_{0}$ be the largest positive integer such that $d_{1} \lambda_{i}<a_{11}$. 
Clearly, if (11) is satisfied, then $1 \leq i_{0}<\infty$. In this case, let

$$
\bar{d}_{2}=\min _{0 \leq i \leq i_{0}} d_{2}^{i}\left(E^{*}\right)
$$

where $d_{2}^{i}\left(E^{*}\right)$ is given by

$$
d_{2}^{i}\left(E^{*}\right)=\frac{d_{1} a_{22} \lambda_{i}-a_{11} a_{22}+a_{12} a_{21}}{\lambda_{i}\left(d_{1} \lambda_{i}-a_{11}\right)} .
$$

Theorem 2.3 Assume that $\left(\mathbf{H}_{\mathbf{1}}\right)$ holds. Then the following conclusions for the model (3) are true.

(i) If $a_{11}<0$, then $E^{*}$ is locally asymptotically stable.

(ii) Let $a_{11}>0,\left(\mathbf{H}_{21}\right)$ and $m u^{*} v^{*}<\left(u^{*}+\delta s v^{*}\right)\left(b v^{*}+a+u^{*}\right)^{2}+b s m u^{*} v^{*}$ hold.

(ii-1) If $d_{1} \lambda_{1}<a_{11}$ and $0<d_{2}<\bar{d}_{2}$, then $E^{*}$ is locally asymptotically stable.

(ii-2) If $d_{1} \lambda_{1}<a_{11}$ and $d_{2}>\bar{d}_{2}$, then $E^{*}$ is unstable, and hence in the model (3) Turing instability occurs.

Proof Consider the linearization operator of (3) at $E^{*}$

$$
L=\left(\begin{array}{cc}
d_{1} \Delta+a_{11} & a_{12} \\
a_{21} & d_{2} \Delta+a_{22}
\end{array}\right)
$$

Suppose that $\Phi=(\varphi, \psi) \in L^{2}(\Omega) \times L^{2}(\Omega)$ is an eigenfunction of $L$ corresponding to an eigenvalue $\eta$, then

$$
\left(d_{1} \Delta \varphi+\left(a_{11}-\eta\right) \varphi+a_{12} \psi, d_{2} \Delta \psi+\left(a_{22}-\eta\right) \psi+a_{21} \varphi\right)=(0,0) .
$$

Writing $\varphi=\sum_{0 \leq i \leq \infty, 1 \leq j \leq m_{i}} a_{i j} \phi_{i j}, \psi=\sum_{0 \leq i \leq \infty, 1 \leq j \leq m_{i}} b_{i j} \phi_{i j}$, then

$$
\sum_{0 \leq i \leq \infty, 1 \leq j \leq m_{i}} B_{i}\left(\begin{array}{l}
a_{i j} \\
b_{i j}
\end{array}\right) \phi_{i j}=0,
$$

where

$$
B_{i}=\left(\begin{array}{cc}
a_{11}-d_{1} \lambda_{i}-\eta & a_{12} \\
a_{21} & a_{22}-d_{2} \lambda_{i}-\eta
\end{array}\right) .
$$

We easily see that $\eta$ is the eigenvalue of $L$ if and only if $\operatorname{det} B_{i}=0$ for some $i$, which leads to

$$
\eta^{2}+Q_{i} \eta+P_{i}=0
$$

where

$$
\begin{aligned}
& Q_{i}=\left(d_{1}+d_{2}\right) \lambda_{i}-a_{11}-a_{22}, \\
& P_{i}=\lambda_{i}\left(d_{1} \lambda_{i}-a_{11}\right)\left(d_{2}-\frac{d_{1} a_{22} \lambda_{i}-a_{11} a_{22}+a_{12} a_{21}}{\lambda_{i}\left(d_{1} \lambda_{i}-a_{11}\right)}\right) .
\end{aligned}
$$


(i) If $a_{11}<0$, then $Q_{i}>0$ and $P_{i}>0$ for all $i$, which implies that $\operatorname{Re}\left\{\eta_{i}\right\}<0$ for all $i$, where $\eta_{i}$ are the eigenvalues of (14). Therefore, the equilibrium point $E^{*}$ is locally asymptotically stable.

(ii) If $\left(\mathbf{H}_{21}\right)$ and $m u^{*} v^{*}<\left(u^{*}+\delta s v^{*}\right)\left(b v^{*}+a+u^{*}\right)^{2}+b s m u^{*} v^{*}$ hold, then $Q_{i}>0$ and $d_{1} a_{22} \lambda_{i}-a_{11} a_{22}+a_{12} a_{21}<0$.

(ii-1) If $a_{11}>0, d_{1} \lambda_{1}<a_{11}$ and $0<d_{2}<\bar{d}_{2}$, then $d_{1} \lambda_{i}<a_{11}$ and $d_{2}<d_{2}^{i}$ for all $i \in\left[1, i_{0}\right]$. Thus,

$$
P_{i}=\lambda_{i}\left(d_{1} \lambda_{i}-a_{11}\right)\left\{d_{2}-\frac{d_{1} a_{22} \lambda_{i}-a_{11} a_{22}+a_{12} a_{21}}{\lambda_{i}\left(d_{1} \lambda_{i}-a_{11}\right)}\right\}>0 .
$$

On the other hand, if $i>i_{0}$, then $d_{1} \lambda_{i}>a_{11}$, and $P_{i}>0$. The analysis yields the locally asymptotical stability of $E^{*}$.

(ii-2) If $a_{11}>0, d_{1} \lambda_{1}<a_{11}$ and $d_{2}>\bar{d}_{2}$, then we may assume the minimum in (13) is attained at $j \in\left[1, i_{0}\right]$. Thus $d_{2}>d_{2}^{j}$, which implies

$$
P_{j}=\lambda_{j}\left(d_{1} \lambda_{j}-a_{11}\right)\left\{d_{2}-\frac{d_{1} a_{22} \lambda_{j}-a_{11} a_{22}+a_{12} a_{21}}{\lambda_{j}\left(d_{1} \lambda_{j}-a_{11}\right)}\right\}<0 .
$$

Hence, $E^{*}$ is unstable in this case. The proof of Theorem 2.3 is complete.

Example 2 We take the parameters in model (2) and (3) as

$$
a=0.1, \quad b=0.2, \quad m=0.6, \quad s=2, \quad q=0.25, \quad \delta=0.1 .
$$

It is easy to verify that there is a unique positive equilibrium point $E^{*}\left(u^{*}, v^{*}\right)=(0.22,0.56)$.

For the ODE model (2), from Theorem 2.2, we can verify that $E^{*}$ is stable. For the PDE model (3) in one-dimensional interval $(0, \pi)$, after fixing $d_{1}=0.015$, from Theorem 2.3, we know that if $d_{2}>\bar{d}_{2}=0.31$, then $E^{*}$ is Turing unstable, and model (3) exhibits Turing pattern. In Fig. 1, we show the numerical results of model (3) with different values for $d_{2}$. Figure 1(a) shows the numerical simulations of Turing instability in model (3) with $d_{2}=$ $0.75>\bar{d}_{2}$. And Fig. 1(b) is for the numerical simulations of the stable positive equilibrium point of model (3) with $d_{2}=0.20<\bar{d}_{2}$. From Fig. 2 we can observe the Turing patterns for the different values of $d_{2}$. One can see that the model exhibits pattern formation, including a cold spots pattern in Fig. 2(a) and a spot-stripe pattern in Fig. 2(b).

\section{Nonexistence/existence of nonconstant positive steady state}

In this section, we consider the nonexistence/existence of nonconstant positive steady states of (4).

Let $N\left(\lambda_{i}\right)$ be the eigenspace corresponding to $\lambda_{i}$ in $H^{1}(\Omega)$. Let $X=\left[H^{1}(\Omega)\right]^{2},\left\{\phi_{i j} ; j=\right.$ $\left.1, \ldots, \operatorname{dim} N\left(\lambda_{i}\right)\right\}$ be an orthonormal basis of $N\left(\lambda_{i}\right)$, and $X_{i j}=\left\{c \Phi_{i j}: c \in R^{2}\right\}$. Then we decompose $X$ as

$$
X=\bigoplus_{i=1}^{\infty} X_{i}, \quad X_{i}=\bigoplus_{j=1}^{\operatorname{dim} N\left(\lambda_{i}\right)} X_{i j}
$$



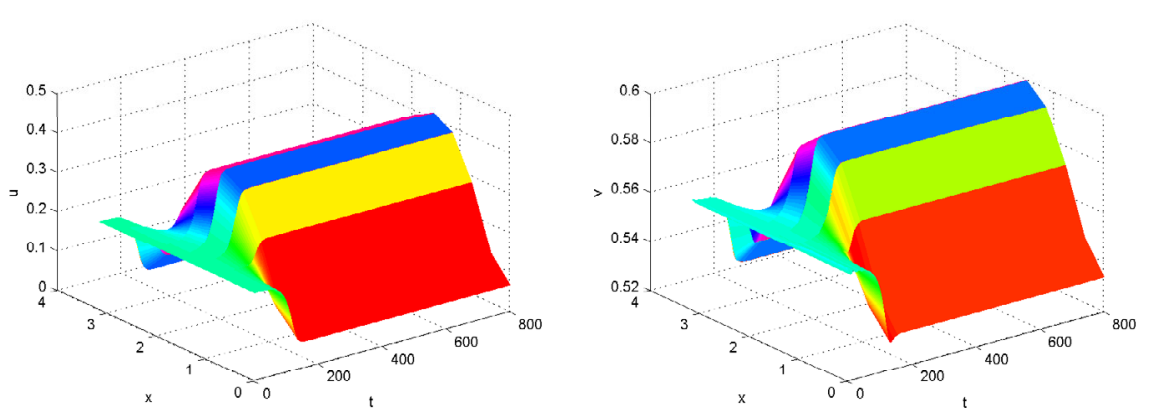

(a) Turing instability with $d_{2}=0.75$
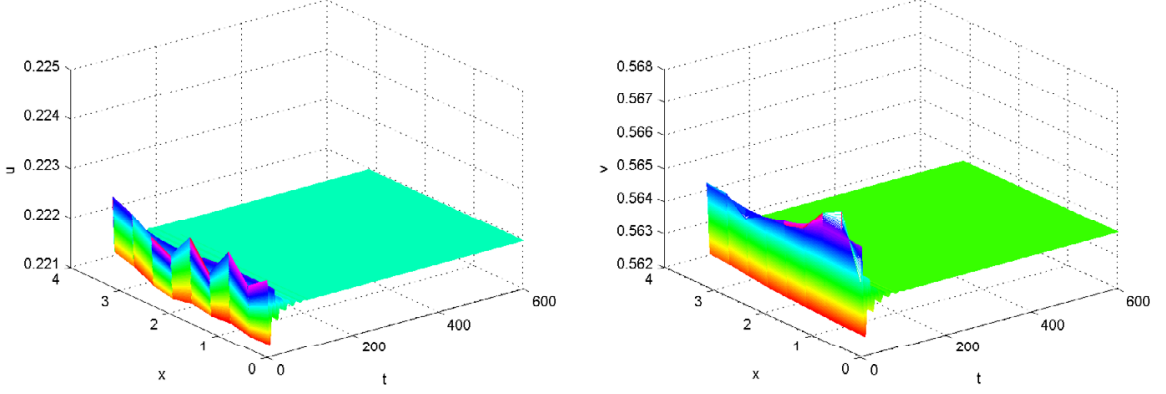

(b) Stable behavior with $d_{2}=0.2$

Figure 1 Numerical simulations of the long time behavior of solution $(u(x, t), v(x, t))$ of model (3) with different values of $d_{2}$. (a) $d_{2}=0.75$; (b) $d_{2}=0.20$

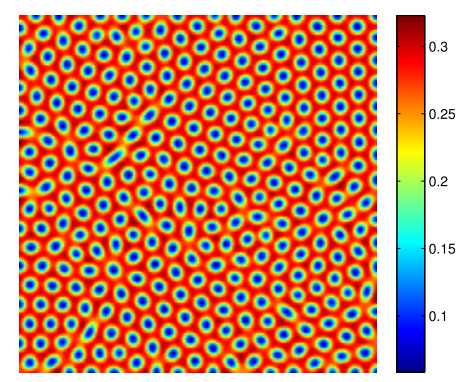

(a) $d_{2}=0.35$

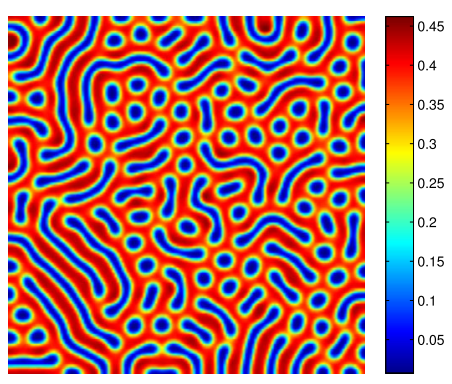

(b) $d_{2}=1$

Figure 2 Turing pattern with different values of $d_{2}$. (a) Cold spots pattern with $d_{2}=0.35$; (b) spot-stripe pattern with $d_{2}=1$

\subsection{A priori estimates for positive steady states}

In this subsection, by using the maximum principle, we establish a priori estimates of positive steady state for (4).

Lemma 3 (Maximum principle [23]) Suppose that $g \in C(\bar{\Omega} \times \mathbb{R})$.

(i) Assume that $w \in C^{2}(\Omega) \cap C^{1}(\bar{\Omega})$, and

$$
\triangle w(x)+g(x, w(x)) \geq 0, \quad x \in \Omega, \quad \frac{\partial w}{\partial v} \leq 0, \quad x \in \partial \Omega .
$$




$$
\text { If } w\left(x_{0}\right)=\max _{\bar{\Omega}} w \text {, then } g\left(x_{0}, w\left(x_{0}\right)\right) \geq 0 .
$$

(ii) Assume that $w \in C^{2}(\Omega) \cap C^{1}(\bar{\Omega})$, and

$$
\begin{gathered}
\Delta w(x)+g(x, w(x)) \leq 0, \quad x \in \Omega, \quad \frac{\partial w}{\partial v} \geq 0, \quad x \in \partial \Omega . \\
\text { If } w\left(x_{0}\right)=\min _{\bar{\Omega}} w, \text { then } g\left(x_{0}, w\left(x_{0}\right)\right) \leq 0 .
\end{gathered}
$$

Theorem 3.1 Assume $m>(a+1) q$. Let $(u(x), v(x))$ be a positive solution of $(4)$. If

$$
a \delta(a+1)>\max \left\{m(m-(a+1) q), m^{2}+a q-m\right\},
$$

then $(u(x), v(x))$ satisfies

$$
1-\frac{m \alpha}{a} \leq u(x) \leq 1, \quad \frac{1}{\delta}\left(\frac{m(a-m \alpha)}{a^{2}+a+(a-m) \alpha}-q\right) \leq v(x) \leq \alpha,
$$

where $\alpha=\frac{m-(a+1) q}{\delta(a+1)}$.

Proof A direct application of Lemma 3 to (4) yields $u(x) \leq 1$ and $v(x) \leq \alpha$. To obtain the lower bound for $u(x)$ and $v(x)$, we let

$$
u\left(x_{0}\right)=\min _{\bar{\Omega}} u(x), \quad v\left(y_{0}\right)=\min _{\bar{\Omega}} v(x), \quad v\left(y_{1}\right)=\max _{\bar{\Omega}} v(x) .
$$

By virtue of Lemma 3, we have

$$
1-u\left(x_{0}\right)-\frac{m \alpha}{a} \leq 1-u\left(x_{0}\right)-\frac{m v\left(x_{0}\right)}{a+u\left(x_{0}\right)+b v\left(x_{0}\right)} \leq 0 .
$$

Since $a \delta(a+1)>m(m-(a+1) q), 1-\frac{m \alpha}{a}>0$ and $u\left(x_{0}\right) \geq 1-\frac{m \alpha}{a}$.

Notice that

$$
-q-\delta v\left(y_{0}\right)+\frac{m u\left(x_{0}\right)}{a+u\left(x_{0}\right)+b v\left(y_{1}\right)} \leq-q-\delta v\left(y_{0}\right)+\frac{m u\left(x_{0}\right)}{a+u\left(x_{0}\right)+b v\left(y_{0}\right)} \leq 0,
$$

we have $v\left(y_{0}\right) \geq \frac{1}{\delta}\left(\frac{m(a-m \alpha)}{a(a+1+\alpha)-m \alpha}-q\right)$. The proof is complete.

\subsection{Nonexistence of nonconstant positive steady state}

In this subsection, we apply the energy method to prove the nonexistence of the nonconstant positive steady state to (4). For convenience, let $\Gamma=\Gamma(m, a, b, s, q, \delta)$ be the set of parameters $m, a, b, s, q$, and $\delta$.

Theorem 3.2 Assume $m>q$. Let $\lambda_{1}$ be the smallest positive eigenvalue of the operator $-\triangle$ on $\Omega$ with zero-flux boundary condition and $d_{2}^{*}$ be a fixed positive constant satisfying $d_{2}^{*}>$ $\frac{s(m-q)}{\lambda_{1}}$. Then there exists a positive $d_{1}^{*}=d_{1}^{*}\left(\Gamma, d_{2}^{*}\right)$ such that model (4) has no nonconstant positive steady state provided that $d_{1} \geq d_{1}^{*}, d_{2} \geq d_{2}^{*}$.

Proof Let $(u, v)$ be a positive solution of (4) and denote

$$
\bar{u}=\frac{1}{|\Omega|} \int_{\Omega} u d x \quad \text { and } \quad \bar{v}=\frac{1}{|\Omega|} \int_{\Omega} v d x .
$$


Then multiplying the first equation of model (4) by $(u-\bar{u})$, integrating over $\Omega$ and from Theorem 3.1, we have

$$
\begin{aligned}
d_{1} \int_{\Omega}|\nabla(u-\bar{u})|^{2} d x= & \int_{\Omega}(u-\bar{u})^{2}\left(1-(u+\bar{u})-\frac{m \bar{v}(a+b v)}{(a+u+b v)(a+\bar{u}+b \bar{v})}\right) d x \\
& -\int_{\Omega} \frac{m \bar{u}(a+u)}{(a+u+b v)(a+\bar{u}+b \bar{v})}(u-\bar{u})(v-\bar{v}) d x \\
\leq & \int_{\Omega}(u-\bar{u})^{2}+m \int_{\Omega}|u-\bar{u}||v-\bar{v}| d x
\end{aligned}
$$

in a similar manner, multiplying the second equation in model (4) by $(v-\bar{v})$, we have

$$
\begin{aligned}
d_{2} \int_{\Omega}|\nabla(v-\bar{v})|^{2} d x= & \int_{\Omega} s(v-\bar{v})^{2}\left(-q-\delta(v+\bar{v})+\frac{m \bar{u}(a+u)}{(a+u+b v)(a+\bar{u}+b \bar{v})}\right) d x \\
& +\int_{\Omega} \frac{m \bar{v}(a+b v)}{(a+u+b v)(a+\bar{u}+b \bar{v})}(u-\bar{u})(v-\bar{v}) d x \\
\leq & s(m-q) \int_{\Omega}(v-\bar{v})^{2} d x+\frac{m}{b} \int_{\Omega}|u-\bar{u}||v-\bar{v}| d x .
\end{aligned}
$$

It follows from the above and the $\epsilon$-Young inequality that

$$
\begin{aligned}
& d_{1} \int_{\Omega}|\nabla(u-\bar{u})|^{2} d x+d_{2} \int_{\Omega}|\nabla(v-\bar{v})|^{2} d x \\
& \quad \leq \int_{\Omega}\left((u-\bar{u})^{2}+s(m-q)(v-\bar{v})^{2}\right) d x+2 L \int_{\Omega}|u-\bar{u}||v-\bar{v}| d x \\
& \quad \leq \int_{\Omega}\left(\left(1+\frac{L}{\epsilon}\right)(u-\bar{u})^{2}+(s(m-q)+\epsilon L)(v-\bar{v})^{2}\right) d x
\end{aligned}
$$

for $L:=\frac{(b+1) m}{2 b}$ and an arbitrary positive constant $\epsilon$. It follows from the well-known Poincaré inequality that

$$
\begin{aligned}
& d_{1} \int_{\Omega}|\nabla(u-\bar{u})|^{2} d x+d_{2} \int_{\Omega}|\nabla(v-\bar{v})|^{2} d x \\
& \quad \leq \frac{1}{\lambda_{1}}\left(\left(1+\frac{L}{\epsilon}\right) \int_{\Omega}|\nabla(u-\bar{u})|^{2} d x+(s(m-q)+\epsilon L) \int_{\Omega}|\nabla(v-\bar{v})|^{2} d x\right) .
\end{aligned}
$$

Since $d_{2}^{*} \lambda_{1}>s(m-q)$, from the assumption, we can choose a sufficiently small $\epsilon$ such that

$$
d_{2}^{*} \lambda_{1} \geq s(m-q)+\epsilon L
$$

Finally, by taking $d_{1}^{*}:=\frac{1}{\lambda_{1}}\left(1+\frac{L}{\epsilon}\right)$, one can conclude that $u=\bar{u}$ and $v=\bar{v}$, which asserts our results.

\subsection{Existence of nonconstant positive steady state}

In this subsection, by using the Leray-Schauder degree theory, we discuss the existence of nonconstant positive steady state to (4) when the diffusion coefficients $d_{1}$ and $d_{2}$ vary while the parameters in $\Gamma$ keep fixed. 
For simplicity, define $F=(f, g)^{\top}$, where $f$ and $g$ are given in Sect. 2 . Then the stationary problem of (4) can be written as

$$
\begin{cases}-\triangle E=D^{-1} F(E), & x \in \Omega \\ \frac{\partial E}{\partial v}=0, & x \in \partial \Omega\end{cases}
$$

where $D=\operatorname{diag}\left(d_{1}, d_{2}\right)$. Therefore, $E$ solves (15) if and only if it satisfies

$$
\widehat{f}\left(d_{1}, d_{2} ; E\right):=E-(I-\Delta)^{-1}\left\{D^{-1} F(E)+E\right\}=0 \quad \text { on } X
$$

where $(I-\Delta)^{-1}$ represents the inverse of $I-\Delta$ with homogeneous Neumann boundary condition.

A straightforward computation reveals

$$
D_{E} \widehat{f}\left(d_{1}, d_{2} ; E^{*}\right)=I-(I-\Delta)^{-1}\left(D^{-1} J\left(E^{*}\right)+I\right) \text {. }
$$

For each $X_{i}, \lambda$ is an eigenvalue of $D_{E} \widehat{f}\left(d_{1}, d_{2} ; E^{*}\right)$ on $X_{i}$ if and only if $\lambda\left(1+\lambda_{i}\right)$ is an eigenvalue of the following matrix:

$$
M_{i}:=\lambda_{i} I-D^{-1} J\left(E^{*}\right)=\left(\begin{array}{cc}
\lambda_{i}-d_{1}^{-1} a_{11} & -d_{1}^{-1} a_{12} \\
-d_{2}^{-1} a_{21} & \lambda_{i}-d_{2}^{-1} a_{22}
\end{array}\right) \text {. }
$$

Clearly,

$$
\operatorname{det} M_{i}=d_{1}^{-1} d_{2}^{-1}\left[d_{1} d_{2} \lambda_{i}^{2}-\left(d_{1} a_{22}+d_{2} a_{11}\right) \lambda_{i}+a_{11} a_{22}-a_{12} a_{21}\right],
$$

and $\operatorname{tr} M_{i}=2 \lambda_{i}-d_{1}^{-1} a_{11}-d_{2}^{-1} a_{22}$. Define

$$
\widehat{g}\left(d_{1}, d_{2} ; \lambda\right)=d_{1} d_{2} \lambda^{2}-\left(d_{1} a_{22}+d_{2} a_{11}\right) \lambda+a_{11} a_{22}-a_{12} a_{21}
$$

Then $\widehat{g}\left(d_{1}, d_{2} ; \lambda\right)=d_{1} d_{2} \operatorname{det} M_{i}$. If

$$
\left|d_{1} a_{22}+d_{2} a_{11}\right|>2 \sqrt{d_{1} d_{2}\left(a_{11} a_{22}-a_{12} a_{21}\right)},
$$

then $\widehat{g}\left(d_{1}, d_{2} ; \lambda\right)=0$ has two real roots:

$$
\begin{aligned}
& \lambda_{-}=\frac{d_{2} a_{11}+d_{1} a_{22}-\sqrt{\left(d_{2} a_{11}+d_{1} a_{22}\right)^{2}-4 d_{1} d_{2}\left(a_{11} a_{22}-a_{12} a_{21}\right)}}{2 d_{1} d_{2}}, \\
& \lambda_{+}=\frac{d_{2} a_{11}+d_{1} a_{22}+\sqrt{\left(d_{2} a_{11}+d_{1} a_{22}\right)^{2}-4 d_{1} d_{2}\left(a_{11} a_{22}-a_{12} a_{21}\right)}}{2 d_{1} d_{2}} .
\end{aligned}
$$

Set

$$
\begin{aligned}
& A=A\left(d_{1}, d_{2}\right)=\left\{\lambda: \lambda \geq 0, \lambda_{-}\left(d_{1}, d_{2}\right)<\lambda<\lambda_{+}\left(d_{1}, d_{2}\right)\right\}, \\
& S_{p}=\left\{\lambda_{0}, \lambda_{1}, \lambda_{2}, \ldots\right\},
\end{aligned}
$$

and let $m\left(\lambda_{i}\right)$ be multiplicity of $\lambda_{i}$. In order to calculate the index of $\widehat{f}\left(d_{1}, d_{2} ; \cdot\right)$ at $E^{*}$, we need the following lemma. 
Lemma 4 ([24]) Suppose $\widehat{g}\left(d_{1}, d_{2} ; \lambda_{i}\right) \neq 0$ for all $\lambda_{i} \in S_{p}$. Then

$$
\operatorname{index}\left(\widehat{f}\left(d_{1}, d_{2} ; \cdot\right), E^{*}\right)=(-1)^{\sigma},
$$

where

$$
\sigma= \begin{cases}\sum_{\lambda_{i} \in A \cap S_{p}} m\left(\lambda_{i}\right), & \text { if } A \cap S_{p} \neq \varnothing, \\ 0, & \text { if } A \cap S_{p}=\varnothing .\end{cases}
$$

In particular, if $\widehat{g}\left(d_{1}, d_{2} ; \lambda_{i}\right)>0$ for all $\lambda_{i} \geq 0$, then $\sigma=0$.

By determining the range of $\lambda$ for which $\widehat{g}\left(d_{1}, d_{2} ; \lambda\right)<0$, we have the existence of nonconstant steady state to (4).

Theorem 3.3 Let $d_{1}, \Gamma$ be fixed and $\left(\mathbf{H}_{1}\right),\left(\mathbf{H}_{21}\right), a_{11}>0$ hold. If $\frac{a_{11}}{d_{1}} \in\left(\lambda_{k}, \lambda_{k+1}\right)$ for some $k \geq 1$, and $\sigma_{k}=\sum_{i=1}^{k} m\left(\lambda_{i}\right)$ is odd, then there exists a positive constant $d^{*}$ such that model (4) has at least one nonconstant positive steady state for all $d_{2} \geq d^{*}$.

Proof Notice that if $d_{2}$ is large enough, then (17) and $\lambda_{+}\left(d_{1}, d_{2}\right)>\lambda_{-}\left(d_{1}, d_{2}\right)>0$ hold. Furthermore,

$$
\lim _{d_{2} \rightarrow \infty} \lambda_{+}\left(d_{1}, d_{2}\right)=\frac{a_{11}}{d_{1}}, \quad \lim _{d_{2} \rightarrow \infty} \lambda_{-}\left(d_{1}, d_{2}\right)=0
$$

As $\frac{a_{11}}{d_{1}} \in\left(\lambda_{k}, \lambda_{k+1}\right)$, there exists $d_{0} \gg 1$ such that

$$
\lambda_{+}\left(d_{1}, d_{2}\right) \in\left(\lambda_{k}, \lambda_{k+1}\right), \quad 0<\lambda_{-}\left(d_{1}, d_{2}\right)<\lambda_{1} \quad \text { for all } d_{2} \geq d_{0} .
$$

From Theorem 3.2, we know that there exists $\tilde{d}>d_{0}$ such that (4) with $d_{1}=\tilde{d}$ and $d_{2} \geq \tilde{d}$ has no nonconstant positive steady state. Let $\tilde{d}>0$ be large enough such that $\frac{a_{11}}{d_{1}}<\lambda_{1}$. Then there exists $d^{*}>\tilde{d}$ such that

$$
0<\lambda_{-}\left(d_{1}, d_{2}\right)<\lambda_{+}\left(d_{1}, d_{2}\right)<\lambda_{1} \quad \text { for all } d_{2} \geq d^{*} .
$$

Now we prove that, for any $d_{2} \geq d^{*}$, (4) has at least one nonconstant positive steady state. By way of contradiction, assume that the assertion is not true for some $d_{2}^{*} \geq d^{*}$. By using the homotopy argument, we can derive a contradiction in the sequel.

Fixing $d_{2}=d_{2}^{*}$, for $t \in[0,1]$, we define

$$
D(t)=\left(\begin{array}{cc}
t d_{1}+(1-t) \tilde{d} & 0 \\
0 & t d_{2}+(1-t) d^{*}
\end{array}\right),
$$

and consider the following problem:

$$
\begin{cases}-\triangle E=D^{-1}(t) F(E), & x \in \Omega, \\ \frac{\partial E}{\partial v}=0, & x \in \partial \Omega .\end{cases}
$$


Notice that $E$ is a nonconstant positive steady state of (4) if and only if it solves (20) with $t=1$. Evidently, $E^{*}$ is the unique constant positive steady state of (20). For any $t \in[0,1], E$ is a nonconstant positive steady state of (20) if and only if it is a solution of the following problem:

$$
h(E ; t)=E-(I-\Delta)^{-1}\left\{D^{-1}(t) F(E)+E\right\}=0 \quad \text { on } X .
$$

Form the above discussion, we know that (21) has no nonconstant positive steady state when $t=0$, and there is no such solution for $t=1$ at $d_{2}=d_{2}^{*}$. Clearly, $h(E ; 1)=\widehat{f}\left(d_{1}, d_{2} ; E\right)$, $h(E ; 0)=\widehat{f}\left(\tilde{d}, d^{*} ; E\right)$ and

$$
\begin{aligned}
& D_{E} \widehat{f}\left(d_{1}, d_{2} ; E^{*}\right)=I-(I-\Delta)^{-1}\left(D^{-1} J\left(E^{*}\right)+I\right), \\
& D_{E} \widehat{f}\left(\tilde{d}, d^{*} ; E^{*}\right)=I-(I-\Delta)^{-1}\left(\widetilde{D}^{-1} J\left(E^{*}\right)+I\right) .
\end{aligned}
$$

Here, $\widehat{f}(\cdot, \cdot ; \cdot)$ is as given in (16) and $\widetilde{D}=\operatorname{diag}\left(\tilde{d}, d^{*}\right)$. Form (18) and (19), we have $A\left(d_{1}, d_{2}\right) \cap$ $S_{p}=\left\{\lambda_{1}, \lambda_{2}, \ldots, \lambda_{k}\right\}$ and $A\left(\tilde{d}, d^{*}\right) \cap S_{p}=\varnothing$. Since $\sigma_{k}$ is odd, Lemma 4 yields

$$
\begin{aligned}
& \left.h\left((\cdot ; 1), E^{*}\right)=\widehat{f}\left(d_{1}, d_{2} ; \cdot\right), E^{*}\right)=(-1)^{\sigma_{k}}=-1, \\
& \left.h\left((\cdot ; 0), E^{*}\right)=\widehat{f}\left(\tilde{d}, d^{*} ; \cdot\right), E^{*}\right)=(-1)^{0}=1 .
\end{aligned}
$$

From Theorem 3.2, there exist positive constants $\underline{C}=\underline{C}\left(\tilde{d}, d_{1}, d^{*}, d_{2}^{*}, \Gamma\right)$ and $\bar{C}=\bar{C}(\tilde{d}$, $\left.d^{*}, \Gamma\right)$ such that the positive solutions of (21) satisfy $\underline{C}<u(x), v(x)<\bar{C}$ on $\bar{\Omega}$ for all $t \in[0,1]$.

Define $\Sigma=\{E \in X: \underline{C}<u(x), v(x)<\bar{C}, x \in \bar{\Omega}\}$. Then $h(E ; t) \neq 0$ for all $E \in \partial \Sigma$ and $t \in$ $[0,1]$. By virtue of the homotopy invariance of the Leray-Schauder degree, we have

$$
\operatorname{deg}(h(\cdot ; 0), \Sigma, 0)=\operatorname{deg}(h(\cdot ; 1), \Sigma, 0) .
$$

Note that both equations $h(E ; 0)=0$ and $h(E ; 1)=1$ have the unique positive solution $E^{*}$ in $\Sigma$, and we obtain

$$
\begin{aligned}
& \operatorname{deg}(h(\cdot ; 0), \Sigma, 0)=\left(h(\cdot ; 0), E^{*}\right)=1, \\
& \operatorname{deg}(h(\cdot ; 1), \Sigma, 0)=\left(h(\cdot ; 1), E^{*}\right)=-1,
\end{aligned}
$$

which contradicts (22). The proof is complete.

\section{Structure of nonconstant positive steady state}

Let $Y=C(\bar{\Omega}) \times C(\bar{\Omega}), X=\left\{(u, v) \mid u, v \in C^{2}(\bar{\Omega}), \frac{\partial u}{\partial v}=\frac{\partial v}{\partial v}=0, x \in \partial \Omega\right\}$.

\subsection{Local structure and direction of nonconstant positive steady state}

In this subsection, we first study the local structure of nonconstant positive steady state for model (4). In brief, by regarding $d_{2}$ as the bifurcation parameter, we verify the existence of positive steady state bifurcating from $\left(d_{2}, E^{*}\right)$. The Crandall-Rabinowitz bifurcation theorem in [25] will be applied to obtain bifurcations.

Define the $\operatorname{map} F:(0, \infty) \times X \rightarrow Y$ by

$$
F\left(d_{2}, E\right)=\left(d_{1} \Delta u+f, d_{2} \Delta v+g\right)^{\top}, \quad E=(u, v),
$$


where $f, g$ are given in Sect. 2. Then the solutions of boundary value problem (4) are exactly zeros of $F$. With $E^{*}=\left(u^{*}, v^{*}\right)$, we have

$$
F\left(d_{2}, E^{*}\right)=0, \quad \text { for all } d_{2}>0 .
$$

If there is a number $d_{2}>0$ such that every neighborhood of $\left(d_{2}, E^{*}\right)$ contains zero of $F$ in $(0, \infty) \times X$ not lying on the curve $\left(d_{2}, E^{*}\right)$, then we say that $\left(d_{2}, E^{*}\right)$ is a bifurcation point of the equation $F=0$ with respect to this curve.

Theorem 4.1 Let $\left(\mathbf{H}_{1}\right),\left(\mathbf{H}_{21}\right)$ and $a_{11}>0$ hold. Suppose that $j$ is a positive integer such that $d_{1} \lambda_{j}<a_{11}$ and $d_{2}^{k} \neq d_{2}^{j}>0$ for any integer $k \neq j$. Then $\left(d_{2}^{j}, E^{*}\right)$ is a bifurcation point of $F\left(d_{2}, E\right)=0$ with respect to the curve $\left(d_{2}, E^{*}\right)$. There is a one-parameter family of non-trivial solution $\Gamma_{j}(s)=\left(d_{2}(s), u(s), v(s)\right)$ of the problem (4) for $|s|$ sufficiently small, where $d_{2}(s), u(s)$, $v(s)$ are continuous functions, $d_{2}(0)=d_{2}^{j}$ and

$$
u(s)=u^{*}+s \phi_{j}+o(s), \quad v(s)=v^{*}+s b_{j} \phi_{j}+o(s), \quad b_{j}=\frac{\left(d_{1} \lambda_{j}-a_{11}\right)}{a_{12}}>0 .
$$

The zero set of $F$ consists of two curves $\left(d_{2}, E^{*}\right)$ and $\Gamma_{j}(s)$ in a neighborhood of the bifurcation point $\left(d_{2}^{j}, E^{*}\right)$.

Proof It suffices to verify conditions (a)-(c) as follows [25]:

(a) The partial derivatives $F_{d_{2}}, F_{E}$, and $F_{d_{2} E}$ exist and are continuous.

(b) $\operatorname{ker} F_{E}\left(d_{2}^{j}, E^{*}\right)$ and $Y / R\left(F_{E}\left(d_{2}^{j}, E^{*}\right)\right)$ are one-dimensional.

(c) Let $\operatorname{ker} F_{E}\left(d_{2}^{j}, E^{*}\right)=\operatorname{span}\{\Phi\}$, then $F_{d_{2} E}\left(d_{2}^{j}, E^{*}\right) \Phi \notin R\left(F_{E}\left(d_{2}^{j}, E^{*}\right)\right)$.

Note that

$$
L_{1}=F_{E}\left(d_{2}^{j}, E^{*}\right)=\left(\begin{array}{cc}
d_{1} \Delta+a_{11} & a_{12} \\
a_{21} & d_{2}^{j} \Delta+a_{22}
\end{array}\right),
$$

where $a_{11}, a_{12}, a_{21}$ and $a_{22}$ are given in (8). It is clear that the linear operators $F_{E}, F_{d_{2} E}$ and $F_{d_{2}}$ are continuous, and condition (a) is verified.

Suppose $\Phi=(\bar{\varphi}, \bar{\psi})^{\top} \in \operatorname{ker} L_{1}$, and write $\bar{\varphi}=\sum_{0 \leq i \leq \infty, 1 \leq j \leq m_{i}} \bar{a}_{i j} \phi_{i j}, \bar{\psi}=\sum_{0 \leq i \leq \infty, 1 \leq j \leq m_{i}} \bar{b}_{i j} \times$ $\phi_{i j}$. Then

$$
\sum_{0 \leq i<\infty, 1 \leq j \leq m_{i}} \bar{B}_{i}\left(\begin{array}{l}
\bar{a}_{i j} \\
\bar{b}_{i j}
\end{array}\right) \phi_{i j}=0,
$$

where

$$
\bar{B}_{i}=\left(\begin{array}{cc}
a_{11}-d_{1} \lambda_{i} & a_{12} \\
a_{21} & a_{22}-d_{2}^{j} \lambda_{i}
\end{array}\right) .
$$

Since

$$
\operatorname{det} \bar{B}_{i}=0 \quad \Leftrightarrow \quad d_{2}^{j}=d_{2}^{i}\left(E^{*}\right)=\frac{d_{1} a_{22} \lambda_{i}-a_{11} a_{22}+a_{12} a_{21}}{\lambda_{i}\left(d_{1} \lambda_{i}-a_{11}\right)},
$$


taking $d_{2}=d_{2}^{j}$ implies that $\operatorname{ker} L_{1}=\operatorname{span}\left\{\Phi_{1}\right\}$, where

$$
\Phi_{1}=\left(1, b_{j}\right)^{\top} \phi_{j}, \quad b_{j}=\frac{d_{1} \lambda_{j}-a_{11}}{a_{12}}>0,
$$

$\phi_{j}$ is the eigenfunction of $-\triangle$. Consider the adjoint operator

$$
L_{1}^{*}=\left(\begin{array}{cc}
d_{1} \Delta+a_{11} & a_{21} \\
a_{12} & d_{2}^{j} \Delta+a_{22}
\end{array}\right) .
$$

In the same way as above we obtain $\operatorname{ker} L_{1}^{*}=\operatorname{span}\left\{\Phi_{1}^{*}\right\}$, where

$$
\Phi_{1}^{*}=\left(1, b_{j}^{*}\right)^{\top} \phi_{j}, \quad b_{j}^{*}=\frac{d_{1} \lambda_{j}-a_{11}}{a_{21}}<0 .
$$

By the Fredholm alternative theorem, we have $R\left(L_{1}\right)=\operatorname{ker}\left(L_{1}^{*}\right)^{\perp}$, thus

$$
\operatorname{codim}\left(R\left(L_{1}\right)\right)=\operatorname{dim}\left(\operatorname{ker}\left(L_{1}^{*}\right)\right)=1 .
$$

Condition (b) is also verified.

Finally, since

$$
F_{d_{2} E}\left(d_{2}^{j}, E^{*}\right) \Phi_{1}=\left(\begin{array}{cc}
0 & 0 \\
0 & \Delta
\end{array}\right) \Phi_{1}=\left(\begin{array}{c}
0 \\
-\lambda_{j} b_{j} \phi_{j}
\end{array}\right)
$$

and

$$
\left\langle F_{d_{2} E}\left(d_{2}^{j}, E^{*}\right) \Phi_{1}, \Phi_{1}^{*}\right\rangle_{Y}=\left\langle-\lambda_{j} b_{j} \phi_{j}, b_{j}^{*} \phi_{j}\right\rangle_{L^{2}}=-\lambda_{j} b_{j} b_{j}^{*}>0,
$$

we find $F_{d_{2} E}\left(d_{2}^{j}, E^{*}\right) \Phi_{1} \notin R\left(L_{1}\right)$, and so condition (c) is satisfied. The proof is completed.

We investigate the direction of the steady state bifurcation of model (4) in the onedimensional interval $\Omega=(0, \pi)$. It is well known that the operator $-\triangle$ with no-flux boundary conditions has eigenvalues and eigenfunctions as follows:

$$
\lambda_{0}=0, \quad \phi_{0}(x)=\sqrt{\frac{1}{\pi}} ; \quad \lambda_{j}=j^{2}, \quad \phi_{j}(x)=\sqrt{\frac{2}{\pi}} \cos j x
$$

for $j=1,2,3, \ldots$ We translate $\left(u^{*}, v^{*}\right)$ to the origin by the translation $(\bar{u}, \bar{v})=\left(u-u^{*}, v-v^{*}\right)$. For convenience, we will denote $\bar{u}, \bar{v}$ by $u, v$, respectively. Then we can obtain the following system:

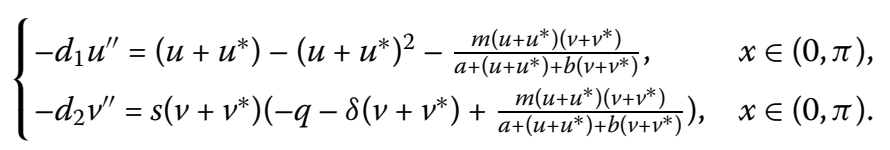

Let

$$
\begin{aligned}
& H=\left(u+u^{*}\right)-\left(u+u^{*}\right)^{2}-\frac{m\left(u+u^{*}\right)\left(v+v^{*}\right)}{a+\left(u+u^{*}\right)+b\left(v+v^{*}\right)}, \\
& G=s\left(v+v^{*}\right)\left(-q-\delta\left(v+v^{*}\right)+\frac{m\left(u+u^{*}\right)\left(v+v^{*}\right)}{a+\left(u+u^{*}\right)+b\left(v+v^{*}\right)}\right) .
\end{aligned}
$$


Then a straightforward calculation yields

$$
\begin{aligned}
& H_{u}(0,0)=1-2 u^{*}-\frac{m v^{*}\left(b v^{*}+a\right)}{\left(b v^{*}+a+u^{*}\right)^{2}}, \quad G_{u}(0,0)=\frac{s m v^{*}\left(b v^{*}+a\right)}{\left(b v^{*}+a+u^{*}\right)^{2}}, \\
& H_{v}(0,0)=-\frac{m v^{*}\left(b v^{*}+a\right)}{\left(b v^{*}+a+u^{*}\right)^{2}}, \quad G_{v}(0,0)=s v\left(-\delta-\frac{m b u^{*}}{\left(b v^{*}+a+u^{*}\right)^{2}}\right), \\
& H_{u u}(0,0)=-2+\frac{2 m v^{*}\left(b v^{*}+a\right)}{\left(b v^{*}+a+u^{*}\right)^{3}}, \quad G_{u u}(0,0)=-\frac{2 s m v^{*}\left(b v^{*}+a\right)}{\left(b v^{*}+a+u^{*}\right)^{3}}, \\
& H_{u v}(0,0)=-\frac{m\left(a^{2}+\left(b v^{*}+u^{*}\right) a+2 b u^{*} v^{*}\right)}{\left(b v^{*}+a+u^{*}\right)^{3}}, \\
& G_{u v}(0,0)=\frac{s m\left(a b v^{*}+2 b u^{*} v^{*}+a^{2}+a u^{*}\right)}{\left(b v^{*}+a+u^{*}\right)^{3}}, \\
& H_{v v}(0,0)=\frac{2 m b u^{*}\left(a+u^{*}\right)}{\left(b v^{*}+a+u^{*}\right)^{3}}, \quad G_{v v}(0,0)=-\frac{2 s\left(\left(b v^{*}+a+u^{*}\right)^{3} \delta+m b u^{*}\left(a+u^{*}\right)\right)}{\left(b v^{*}+a+u^{*}\right)^{3}}, \\
& H_{u u u}(0,0)=-\frac{6 m v^{*}\left(b v^{*}+a\right)}{\left(b v^{*}+a+u^{*}\right)^{4}}, \quad G_{u u u}(0,0)=\frac{6 s m v^{*}\left(b v^{*}+a\right)}{\left(b v^{*}+a+u^{*}\right)^{4}}, \\
& H_{u u v}(0,0)=\frac{2 m\left(-b^{2} v^{* 2}+2 b u^{*} v^{*}+a^{2}+a u^{*}\right)}{\left(b v^{*}+a+u^{*}\right)^{4}}, \\
& G_{u u v}(0,0)=-\frac{2 s m\left(-b^{2} v^{* 2}+2 b u^{*} v^{*}+a^{2}+a u^{*}\right)}{\left(b v^{*}+a+u^{*}\right)^{4}}, \\
& H_{u v v}(0,0)=\frac{2 m b\left(a b v^{*}+2 b u^{*} v^{*}+a^{2}-u^{* 2}\right)}{\left(b v^{*}+a+u^{*}\right)^{4}}, \\
& G_{u v v}(0,0)=-\frac{2 s m b\left(a b v^{*}+2 b u^{*} v^{*}+a^{2}-u^{* 2}\right)}{\left(b v^{*}+a+u^{*}\right)^{4}}, \\
& H_{v v v}(0,0)=-\frac{6 m b^{2} u^{*}\left(a+u^{*}\right)}{\left(b v^{*}+a+u^{*}\right)^{4}}, \quad G_{v v v}(0,0)=\frac{6 s m b^{2} u^{*}\left(a+u^{*}\right)}{\left(b v^{*}+a+u^{*}\right)^{4}} .
\end{aligned}
$$

Denote $E=(u, v)$, then we rewrite the map $F: \mathbb{R}^{+} \times X \rightarrow Y$ by

$$
F\left(d_{2}, E\right)=\left(\begin{array}{l}
d_{1} u^{\prime \prime}+H(u, v) \\
d_{2} v^{\prime \prime}+G(u, v)
\end{array}\right) .
$$

By Theorem 4.1, we see that $\operatorname{dimker} F_{E}\left(d_{2}^{j},(0,0)\right)=\operatorname{codim} R\left(F_{E}\left(d_{2}^{j},(0,0)\right)\right)=1$ and $\operatorname{ker} F_{E}\left(d_{2}^{j}\right.$, $(0,0))=\operatorname{span}\left\{\Phi_{1}\right\}$. Hence, we can decompose $X$ and $Y$ as

$$
X=\operatorname{ker} F_{E}\left(d_{2}^{j},(0,0)\right) \oplus Z \quad \text { and } \quad Y=R\left(F_{E}\left(d_{2}^{j},(0,0)\right)\right) \oplus Z^{\prime},
$$

where $Z$ is the complement of $\operatorname{ker} F_{E}\left(d_{2}^{j},(0,0)\right)$ in $X$ and $Z^{\prime}$ is the complement of $R\left(F_{E}\left(d_{2}^{j},(0,0)\right)\right)$ in $Y$. Due to $\operatorname{codim} R\left(F_{E}\left(d_{2}^{j},(0,0)\right)\right)=1$, there exists $T \in Y^{*}$ such that

$$
R\left(F_{E}\left(d_{2}^{j},(0,0)\right)\right)=\{(\xi, \zeta) \in Y:\langle T,(\xi, \zeta)\rangle=0\},
$$

where $Y^{*}:=\operatorname{span}\left\{\Phi_{1}^{*}\right\}$. Moreover, $\Phi_{1}^{*}$ satisfies $F_{E}\left(d_{2}^{j},(0,0)\right) \Phi_{1}^{*}=0$ by Theorem 4.1. Hence, we can define

$$
\langle T,(\xi, \zeta)\rangle=\left\langle\Phi_{1}^{*},(\xi, \zeta)\right\rangle=\int_{\Omega} \xi \phi_{j} d x+\int_{\Omega} b_{j}^{*} \zeta \phi_{j} d x .
$$


By $F_{d_{2} E}\left(d_{2}^{j},(0,0)\right) \Phi_{1} \notin R\left(F_{E}\left(d_{2}^{j},(0,0)\right)\right)$ derived in Theorem 4.1 , we find that

$$
\left\langle F_{d_{2} E}\left(d_{2}^{j},(0,0)\right) \Phi_{1}, \Phi_{1}^{*}\right\rangle \neq 0
$$

From [26], we can know that

$$
d_{2}^{\prime}(0)=-\frac{\left\langle F_{E E}\left(d_{2}^{j},(0,0)\right) \Phi_{1}^{2}, \Phi_{1}^{*}\right\rangle}{2\left\langle F_{d_{2} E}\left(d_{2}^{j},(0,0)\right) \Phi_{1}, \Phi_{1}^{*}\right\rangle}
$$

By some calculations, we have

$$
\left\langle F_{E E}\left(d_{2}^{j},(0,0)\right) \Phi_{1}^{2}, \Phi_{1}^{*}\right\rangle=\left(g_{j}+h_{j} b_{j}^{*}\right) \int_{0}^{\pi} \phi_{j}^{3} d x=0
$$

and

$$
\left\langle F_{d_{2} E}\left(d_{2}^{j},(0,0)\right) \Phi_{1}, \Phi_{1}^{*}\right\rangle=\int_{0}^{\pi} b_{j}^{*} \phi_{j}\left(b_{j} \phi_{j}\right)^{\prime \prime} d x=-j^{2} b_{j} b_{j}^{*}
$$

where

$$
\begin{gathered}
g_{j}=H_{u u}(0,0)+2 H_{u v}(0,0) b_{j}+H_{v v}(0,0) b_{j}^{2}, \\
h_{j}=G_{u u}(0,0)+2 G_{u v}(0,0) b_{j}+G_{v v}(0,0) b_{j}^{2} .
\end{gathered}
$$

Hence, $d_{2}^{\prime}(0)=0$.

Note that $\left\langle F_{E E}\left(d_{2}^{j},(0,0)\right) \Phi_{1}^{2}, \Phi_{1}^{*}\right\rangle=0$ implies

$$
F_{E E}\left(d_{2}^{j},(0,0)\right) \Phi_{1}^{2} \in R\left(F_{E}\left(d_{2}^{j},(0,0)\right)\right)
$$

From [26], we see that the bifurcation is supercritical (resp. subcritical) if

$$
d^{\prime \prime}(0)=-\frac{\left\langle F_{E E E}\left(d_{2}^{j},(0,0)\right) \Phi_{1}^{3}, \Phi_{1}^{*}\right\rangle+3\left\langle F_{E E}\left(d_{2}^{j},(0,0)\right) \Phi_{1} \theta, \Phi_{1}^{*}\right\rangle}{3\left\langle F_{d_{2} E}\left(d_{2}^{j},(0,0)\right) \Phi_{1}, \Phi_{1}^{*}\right\rangle}>0 \quad(\text { resp. }<0)
$$

where $\theta$ is the solution of the following problem:

$$
F_{E E}\left(d_{2}^{j},(0,0)\right) \Phi_{1}^{2}+F_{E}\left(d_{2}^{j},(0,0)\right) \theta=0
$$

Let $\theta=\left(\theta_{1}, \theta_{2}\right)$. Then $\theta$ satisfies

$$
\left\{\begin{array}{l}
d_{1} \theta_{1}^{\prime \prime}+H_{u}(0,0) \theta_{1}+H_{v}(0,0) \theta_{2}=-g_{j} \phi_{j}^{2} \\
d_{2}^{j} \theta_{2}^{\prime \prime}+G_{u}(0,0) \theta_{1}+G_{v}(0,0) \theta_{2}=-h_{j} \phi_{j}^{2} \\
\theta_{i}^{\prime}(0, t)=\theta_{i}^{\prime}(\pi, t)=0, \quad i=1,2 .
\end{array}\right.
$$

By direct calculation, we obtain

$$
\left\langle F_{E E E}\left(d_{2}^{j},(0,0)\right) \Phi_{1}^{3}, \Phi_{1}^{*}\right\rangle=\left(m_{j}+n_{j} b_{j}^{*}\right) \int_{0}^{\pi} \phi_{j}^{4} d x=\frac{3}{2 \pi}\left(m_{j}+n_{j} b_{j}^{*}\right)
$$


where

$$
\begin{aligned}
& m_{j}=H_{u u u}(0,0)+3 b_{j} H_{u u v}(0,0)+3 b_{j}^{2} H_{u v v}(0,0)+b_{j}^{3} H_{v v v}(0,0), \\
& n_{j}=G_{u u u}(0,0)+3 b_{j} G_{u u v}(0,0)+3 b_{j}^{2} G_{u v v}(0,0)+b_{j}^{3} G_{v v v}(0,0),
\end{aligned}
$$

and $b_{j}, b_{j}^{*}$ are given in Sect. 4.1. Hence,

$$
\begin{aligned}
\left\langle F_{E E E}\right. & \left.\left(d_{2}^{j},(0,0)\right) \Phi_{1}^{3}, \Phi_{1}^{*}\right\rangle \\
= & \frac{3}{2 \pi}\left(3 b_{j}\left[b_{j}^{*} G_{u u v}(0,0)+H_{u u v}(0,0)\right]+3 b_{j}^{2}\left[H_{u v v}(0,0)+b_{j}^{*} G_{u v v}(0,0)\right]\right. \\
& \left.+b_{j}^{3}\left[H_{v v v}(0,0)+b_{j}^{*} G_{v v v}(0,0)\right]+b_{j}^{*} G_{u u u}(0,0)+H_{u u u}(0,0)\right)
\end{aligned}
$$

and

$$
\left\langle F_{E E}\left(d_{2}^{j},(0,0) \Phi_{1} \theta, \Phi_{1}^{*}\right\rangle=C_{1} \int_{0}^{\pi} \theta_{1} \phi_{j}^{2} d x+C_{2} \int_{0}^{\pi} \theta_{2} \phi_{j}^{2} d x,\right.
$$

where

$$
\begin{aligned}
& C_{1}=H_{u u}(0,0)+b_{j} H_{u v}(0,0)+b_{j}^{*} G_{u u}(0,0)+b_{j} b_{j}^{*} G_{u v}(0,0), \\
& C_{2}=H_{u v}(0,0)+b_{j} H_{v v}(0,0)+b_{j}^{*} G_{u v}(0,0)+b_{j} b_{j}^{*} G_{v v}(0,0) .
\end{aligned}
$$

We now compute

$$
\int_{0}^{\pi} \theta_{1} \phi_{j}^{2} d x \text { and } \int_{0}^{\pi} \theta_{2} \phi_{j}^{2} d x
$$

Multiplying (25) by $\phi_{j}^{2}$ and integrating by parts, we derive

$$
\left\{\begin{array}{l}
d_{1} \int_{0}^{\pi} \phi_{j}^{2} \theta_{1}^{\prime \prime} d x+H_{u}(0,0) \int_{0}^{\pi} \phi_{j}^{2} \theta_{1} d x+H_{v}(0,0) \int_{0}^{\pi} \phi_{j}^{2} \theta_{2} d x=-g_{j} \int_{0}^{\pi} \phi_{j}^{4} d x \\
d_{2}^{j} \int_{0}^{\pi} \phi_{j}^{2} \theta_{2}^{\prime \prime} d x+G_{u}(0,0) \int_{0}^{\pi} \phi_{j}^{2} \theta_{1} d x+G_{\nu}(0,0) \int_{0}^{\pi} \phi_{j}^{2} \theta_{2} d x=-h_{j} \int_{0}^{\pi} \phi_{j}^{4} d x
\end{array}\right.
$$

where

$$
\int_{0}^{\pi} \phi_{j}^{2} \theta_{i}^{\prime \prime} d x=\frac{4}{\pi} j^{2} \int_{0}^{\pi} \theta_{i}\left(1-2 \cos ^{2} j x\right) d x, \quad i=1,2 .
$$

Integrating (25) by parts yields

$$
\begin{aligned}
& \gamma_{1}:=\int_{0}^{\pi} \theta_{1} d x=\frac{\left(h_{j} H_{\nu}(0,0)-g_{j} G_{v}(0,0)\right)}{\left(H_{u}(0,0) G_{v}(0,0)-H_{\nu}(0,0) G_{u}(0,0)\right)}, \\
& \gamma_{2}:=\int_{0}^{\pi} \theta_{2} d x=\frac{\left(g_{j} G_{u}(0,0)-h_{j} H_{u}(0,0)\right)}{\left(H_{u}(0,0) G_{v}(0,0)-H_{\nu}(0,0) G_{u}(0,0)\right)} .
\end{aligned}
$$

It follows from (26) that

$$
\left\{\begin{array}{l}
\left(H_{u}(0,0)-4 d_{1} j^{2}\right) \int_{0}^{\pi} \phi_{j}^{2} \theta_{1} d x+H_{v}(0,0) \int_{0}^{\pi} \phi_{j}^{2} \theta_{2} d x=-\frac{3 g_{j}}{2 \pi}-\frac{4}{\pi} d_{1} \gamma_{1} j^{2} \\
\left(G_{v}(0,0)-4 d_{2}^{j} j^{2}\right) \int_{0}^{\pi} \phi_{j}^{2} \theta_{2} d x+G_{u}(0,0) \int_{0}^{\pi} \phi_{j}^{2} \theta_{1} d x=-\frac{3 h_{j}}{2 \pi}-\frac{4}{\pi} d_{2}^{j} \gamma_{2} j^{2}
\end{array}\right.
$$


Thus,

$$
L_{1}:=\int_{0}^{\pi} \theta_{1} \phi_{j}^{2} d x=\frac{A_{1}}{B}, \quad L_{2}:=\int_{0}^{\pi} \theta_{2} \phi_{j}^{2} d x=\frac{A_{2}}{B},
$$

where

$$
\begin{aligned}
& A_{1}:=\left(-\frac{3}{2 \pi} g_{j}-\frac{4}{\pi} d_{1} \gamma_{1} j^{2}\right)\left(G_{\nu}(0,0)-4 d_{2}^{j} j^{2}\right)+H_{\nu}(0,0)\left(\frac{3}{2 \pi} h_{j}+\frac{4}{\pi} d_{2}^{j} \gamma_{2} j^{2}\right), \\
& A_{2}:=\left(-\frac{3}{2 \pi} h_{j}-\frac{4}{\pi} d_{2}^{j} \gamma_{2} j^{2}\right)\left(H_{u}(0,0)-4 d_{1} j^{2}\right)+G_{u}(0,0)\left(\frac{3}{2 \pi} g_{j}+\frac{4}{\pi} d_{1} \gamma_{1} j^{2}\right), \\
& B:=\left(H_{u}(0,0)-4 d_{1} j^{2}\right)\left(G_{v}(0,0)-4 d_{2}^{j} j^{2}\right)-H_{\nu}(0,0) G_{u}(0,0) .
\end{aligned}
$$

Consequently, we have

$$
d_{2}^{\prime \prime}(0)=\frac{C}{2 \pi j^{2} b_{j} b_{j}^{*}},
$$

where

$$
\begin{aligned}
C:= & 3 b_{j}\left[b_{j}^{*} G_{u u v}(0,0)+H_{u u v}(0,0)\right]+3 b_{j}^{2}\left[H_{u v v}(0,0)+b_{j}^{*} G_{u v v}(0,0)\right] \\
& +b_{j}^{3}\left[H_{v v v}(0,0)+b_{j}^{*} G_{v v v}(0,0)\right]+b_{j}^{*} G_{u u u}(0,0)+H_{u u u}(0,0)+2 \pi\left(C_{1} L_{1}+C_{2} L_{2}\right) .
\end{aligned}
$$

From the analysis above, we obtain the following results.

Theorem 4.2 Under the same hypothesis as Theorem 4.1, there exists a smooth bifurcation branch from $\left(d_{2}^{j},(0,0)\right)$. Furthermore, the bifurcation is supercritical (resp. subcritical) provided that $d_{2}^{\prime \prime}(0)>0(<0)$, where $d_{2}^{\prime \prime}(0)$ is given by $(27)$.

\subsection{Global structure of nonconstant positive steady state}

Theorem 4.1 provides no information of the bifurcating curve $\Gamma_{j}$ far from the equilibrium point. A further study is therefore necessary in order to understand its global bifurcation. In the one-dimensional interval $\Omega=(0, \pi)$, by using the global bifurcation theory of Rabinowitz and the Leray-Schauder degree for compact operates, we prove that $\Gamma_{j}$ is unbounded.

Theorem 4.3 Under the same hypothesis as Theorem 4.1, the projection of the bifurcation curve $\Gamma_{j}$ on the $d_{2}$-axis contains $\left(d_{2}^{j}, \infty\right)$.

If $d_{2}>\bar{d}_{2}$ and $d_{2} \neq d_{2}^{k}$ for any integer $k>0$, then the problem (4) possesses at least one nonconstant positive steady state.

Proof Let $\tilde{u}=u-u^{*}, \tilde{v}=v-v^{*}$. Then (4) is transformed into

$$
\left\{\begin{array}{l}
-d_{1} \tilde{u}^{\prime \prime}=a_{11} \tilde{u}+a_{12} \tilde{v}+h_{1}(\tilde{u}, \tilde{v}), \\
-d_{2} \tilde{v}^{\prime \prime}=a_{21} \tilde{u}+a_{22} \tilde{v}+h_{2}(\tilde{v}, \tilde{v}),
\end{array}\right.
$$


where $h_{1}(\tilde{u}, \tilde{v}), h_{2}(\tilde{u}, \tilde{v})$ are higher-order terms of $\tilde{u}$ and $\tilde{v}$. The equilibrium point $\left(u^{*}, v^{*}\right)$ of (4) shifts to $(0,0)$ of this new system. Let

$$
G_{1}=\left(-d_{1} \frac{\partial^{2}}{\partial x^{2}}+a_{11}\right)^{-1}, \quad G_{2}=\left(-d_{2} \frac{\partial^{2}}{\partial x^{2}}-a_{22}\right)^{-1} .
$$

Then (28) is transformed into

$$
\tilde{u}=G_{1}\left(2 a_{11} \tilde{u}\right)+G_{1}\left(a_{12} \tilde{v}\right)+G_{1}\left(h_{1}(\tilde{u}, \tilde{v})\right), \quad \tilde{v}=G_{2}\left(a_{21} \tilde{v}\right)+G_{2}\left(h_{2}(\tilde{u}, \tilde{v})\right) .
$$

Put $\tilde{E}=(\tilde{u}, \tilde{v}), K\left(d_{2}\right) \tilde{E}=\left(2 a_{11} G_{1}(\tilde{u})+a_{12} G_{1}(\tilde{v}), a_{21} G_{2}(\tilde{u})\right)$ and

$$
H(\tilde{E})=\left(G_{1}\left(h_{1}(\tilde{u}, \tilde{v})\right), G_{2}\left(h_{2}(\tilde{u}, \tilde{v})\right) .\right.
$$

Recall that

$$
U=\left\{(u, v): u, v \in C^{2}([0, \pi]), u^{\prime}=v^{\prime}=0 \text { at } x=0, \pi\right\} .
$$

Then the boundary value problem (4) can be interpreted as the equation

$$
\tilde{E}=K\left(d_{2}\right) \tilde{E}+H(\tilde{E}) \quad \text { in } E .
$$

Note that $K\left(d_{2}\right)$ is a compact linear operator on $U$ for any given $d_{2}>0$ and $H(\tilde{E})=o(|\tilde{E}|)$ for $\tilde{E}$ near zero uniformly on closed $d_{2}$ sub-intervals of $(0, \infty)$, and $H(\tilde{E})$ is a compact operator on $U$ as well.

In order to apply Rabinowitz's global bifurcation theorem, we first verify that 1 is an eigenvalue of $K\left(d_{2}^{j}\right)$ of algebraic multiplicity one. From the argument in the proof of Theorem 4.1 it is seen that $\operatorname{ker}\left(K\left(d_{2}^{j}\right)-I\right)=\operatorname{ker} L_{1}=\operatorname{span}\left\{\Phi_{1}\right\}$, so 1 is indeed an eigenvalue of $K=K\left(d_{2}^{j}\right)$, and $\operatorname{dim} \operatorname{ker}(K-I)=1$. As the algebraic multiplicity of the eigenvalue 1 is the dimension of the generalized null space $\bigcup_{i=1}^{\infty} \operatorname{ker}(K-I)^{i}$, we need to verify that $\operatorname{ker}(K-I)=\operatorname{ker}(K-I)^{2}$, or $\operatorname{ker}(K-I) \cap R(K-I)=0$.

We now compute $\operatorname{ker}\left(K^{*}-I\right)$ following the calculation in [27], where $K^{*}$ is the adjoint of $K$. Let $(\hat{\varphi}, \hat{\psi}) \in \operatorname{ker}\left(K^{*}-I\right)$. Then

$$
2 a_{11} G_{1}(\hat{\varphi})+a_{21} G_{2}(\hat{\psi})=\hat{\varphi}, \quad a_{12} G_{1}(\hat{\varphi})=\hat{\psi} .
$$

By the definition of $G_{1}$ and $G_{2}$ we obtain

$$
-d_{2}^{j} a_{12} \hat{\varphi}^{\prime \prime}=f_{\hat{\varphi}} \hat{\varphi}+f_{\hat{\psi}} \hat{\psi}, \quad-d_{1} \hat{\psi}^{\prime \prime}=a_{12} \hat{\varphi}-a_{11} \hat{\psi},
$$

where

$$
f_{\hat{\varphi}}=\frac{2 d_{2}^{j} a_{11} a_{12}}{d_{1}}+a_{12} a_{22}, \quad f_{\hat{\psi}}=a_{12} a_{21}-2\left(a_{11} a_{22}+\frac{d_{2}^{j} a_{11}^{2}}{d_{1}}\right) .
$$

Write $\hat{\varphi}=\sum_{0 \leq i \leq \infty, 1 \leq j \leq m_{i}} \hat{a}_{i j} \phi_{i j}, \hat{\psi}=\sum_{0 \leq i \leq \infty, 1 \leq j \leq m_{i}} \hat{b}_{i j} \phi_{i j}$. Then

$$
\sum_{0 \leq i \leq \infty, 1 \leq j \leq m_{i}} \hat{B}_{i}\left(\begin{array}{l}
\hat{a}_{i j} \\
\hat{b}_{i j}
\end{array}\right) \phi_{i j}=0,
$$


where

$$
\hat{B}_{i}=\left(\begin{array}{cc}
-d_{2}^{j} a_{12} \lambda_{i}+f_{\hat{\varphi}} & f_{\hat{\psi}} \\
a_{12} & -d_{1} \lambda_{i}-a_{11}
\end{array}\right) .
$$

By a straightforward calculation one can check that $\operatorname{det} \hat{B}_{i}=a_{12} \operatorname{det} \bar{B}_{i}$, where $\bar{B}_{i}$ is given in (23) by replacing $d_{2}$ with $d_{2}^{j}$. Thus $\operatorname{det} \bar{B}_{i}=0$ only for $i=j$, and $\operatorname{ker}\left(K^{*}-I\right)=\operatorname{span}\{\hat{\Phi}\}$, where $\hat{\Phi}=\left(\frac{d_{1} \lambda_{i}+a_{11}}{a_{12}}, 1\right)^{\top} \phi_{j}$. Since $\left(\Phi_{1}, \hat{\Phi}\right)_{Y}=\frac{2 d_{1} \lambda_{j}}{a_{12}} \neq 0, \Phi_{1} \notin\left(\operatorname{ker}\left(K^{*}-I\right)\right)^{\perp}=R((K-I))$, so $\operatorname{ker}(K-I) \cap R(K-I)=0$ and the eigenvalue 1 has algebraic multiplicity one.

If $0<d_{2} \neq d_{2}^{j}$ is in a small neighborhood of $d_{2}^{j}$, then the linear operator $I-K\left(d_{2}\right): U \rightarrow U$ is a bijection and 0 is an isolated solution of (29) for this fixed $d_{2}$. The index of this isolated zero of $I-K\left(d_{2}\right)-H$ is given by

$$
\operatorname{index}\left(I-K\left(d_{2}\right)-H,\left(d_{2}, 0\right)\right)=\operatorname{deg}\left(I-K\left(d_{2}\right), B, 0\right)=(-1)^{p},
$$

where $B$ is a sufficiently small ball with center at 0 , and $p$ is the sum of the algebraic multiplicities of the eigenvalues of $K\left(d_{2}\right)$ which are larger than 1 . For our bifurcation analysis, it is also necessary to verify that this index changes as $d_{2}$ crosses $d_{2}^{j}$, that is, for $\epsilon>0$ sufficiently small,

$$
\operatorname{index}\left(I-K\left(d_{2}^{j}-\epsilon\right)-H,\left(d_{2}^{j}-\epsilon, 0\right)\right) \neq \operatorname{index}\left(I-K\left(d_{2}^{j}+\epsilon\right)-H,\left(d_{2}^{j}+\epsilon, 0\right)\right) \text {. }
$$

Indeed, if $\mu$ is an eigenvalue of $K\left(d_{2}\right)$ with an eigenfunction $(\tilde{\varphi}, \tilde{\psi})$, then

$$
2 a_{11} G_{1}(\tilde{\varphi})+a_{12} G_{1}(\tilde{\psi})=\mu \tilde{\varphi}, \quad a_{21} G_{2}(\tilde{\varphi})=\mu \tilde{\psi} .
$$

By the definition of $G_{1}, G_{2}$ and

$$
\tilde{\varphi}=\sum_{0 \leq i \leq \infty, 1 \leq j \leq m_{i}} \tilde{a}_{i j} \phi_{i j}, \tilde{\psi}=\sum_{0 \leq i \leq \infty, 1 \leq j \leq m_{i}} \tilde{b}_{i j} \phi_{i j}
$$

we have

$$
\sum_{0 \leq i \leq \infty, 1 \leq j \leq m_{i}} \tilde{B}_{i}\left(\begin{array}{l}
\tilde{a}_{i j} \\
\tilde{b}_{i j}
\end{array}\right) \phi_{i j}=0,
$$

where

$$
\tilde{B}_{i}=\left(\begin{array}{cc}
(2-\mu) a_{11}-d_{1} \lambda_{i} \mu & a_{12} \\
a_{21} & \left(a_{22}-d_{2} \lambda_{i}\right) \mu
\end{array}\right) .
$$

Thus the set of eigenvalues of $K\left(d_{2}\right)$ consists of all $\mu$ that solve the characteristic equation

$$
\mu^{2}-\frac{2 a_{11}}{d_{1} \lambda_{i}+a_{11}} \mu-\frac{a_{12} a_{21}}{\left(d_{2} \lambda_{i}-a_{22}\right)\left(d_{1} \lambda_{i}+a_{11}\right)}=0 .
$$

In particular, for $d_{2}=d_{2}^{j}$, if $\mu=1$ is a root of (31), then a simple calculation leads to $d_{2}^{j}=d_{2}^{i}$, and $j=i$ by the assumption. For $i=j$ in (31), we let $\mu_{1}\left(d_{2}^{j}\right), \mu_{2}\left(d_{2}^{j}\right)$ denote the two roots. 
First we find that

$$
\mu_{1}\left(d_{2}^{j}\right)=1 \quad \text { and } \quad \mu_{2}\left(d_{2}^{j}\right)=\frac{a_{11}-d_{1} \lambda_{j}}{a_{11}+d_{1} \lambda_{j}}<1
$$

Now for $d_{2}$ close to $d_{2}^{j}$, the root of (31) is given by

$$
\mu_{1}\left(d_{2}\right)=\frac{a_{11}+\sqrt{a_{11}^{2}+\frac{a_{12} a_{21}\left(d_{1} \lambda_{i}+a_{11}\right)}{d_{2} \lambda_{i}-a_{22}}}}{\left(d_{1} \lambda_{i}+a_{11}\right)}, \quad \mu_{2}\left(d_{2}\right)<1 .
$$

And $\mu_{2}\left(d_{2}\right)$ is an increasing function of $d_{2}$, there is a small $\epsilon>0$ such that

$$
\mu_{1}\left(d_{2}^{j}+\epsilon\right)>1, \quad \mu_{1}\left(d_{2}^{j}-\epsilon\right)<1
$$

Consequently, $K\left(d_{2}^{j}+\epsilon\right)$ has exactly one more eigenvalues that are larger than 1 than $K\left(d_{2}^{j}-\right.$ $\epsilon)$ does, and by a similar argument to above we can show this eigenvalue has algebraic multiplicity one. This verifies (30).

With the help of (30), we can use the argument in [25] to conclude that $\Gamma_{j}$ either meets infinity in $R \times U$ or meets $\left(d_{2}^{k}, 0\right)$ for some $k \neq j, d_{2}^{k}>0$. We now show that the first alternative must occur, following the idea of [28] and [29]. Indeed, if $\Gamma_{j}$ is bounded, then it is compact, and $\Gamma_{j}$ meets some other bifurcation points. Let $k$ be such that $\Gamma_{j}$ meets $\left(d_{2}^{k}, 0\right)$, but not $\left(d_{2}^{i}, 0\right)$ for any $i>k$. Consider the problem $(4)$ on the interval $(0, \pi)$ subject to the boundary condition

$$
u^{\prime}=v^{\prime} \quad \text { at } x=0, \pi
$$

We first note that if $\bar{E}$ solves (4) and (32), then one can construct a solution $E$ of (4) by a reflective and periodic extension: Let $x_{n}=n \pi, n=0,1, \ldots, k$, and define

$$
E(x)= \begin{cases}\bar{E}\left(x-x_{2 n}\right) & \text { if } x_{2 n} \leq x \leq x_{2 n+1}, \\ \bar{E}\left(x_{2 n+2}-x\right) & \text { if } x_{2 n+1} \leq x \leq x_{2 n+2}\end{cases}
$$

It is easy see that $\left(d_{2}^{k}, 0\right)$ is also a bifurcation point of the problem (4) and (32). Let $\Lambda_{k}$ denote the bifurcation branch of this new problem that meets infinity or meets $\left(d_{2}^{k}, 0\right)$, then use the same argument above it is clear that it either meets infinity or meets $\left(d_{2}^{k^{\prime}}, 0\right)$ for some $k^{\prime}>k$. If the second case occurs, then by the above extension one sees that $\Gamma_{j}$ meets $\left(d_{2}^{k^{\prime}}, 0\right)$, which violates the definition of $k$, hence $\Lambda_{k}$ meets infinity, and then by the extension again $\Gamma_{j}$ meets infinity too. It then follows that the projection of $\Gamma_{j}$ on the $d_{2}$ interval must be unbounded, since the solutions $u, v$ are bounded by constants independent of $d_{2}$. It also follows from the a priori estimates that any solutions on the curve $\Gamma_{j}$ must be positive. And the proof is complete. 
Availability of data and materials

Data sharing not applicable to this article as no data sets were generated or analyzed during the current study.

\section{Competing interests}

The authors declare that there is no conflict of interests regarding the publication of this paper

\section{Authors' contributions}

All authors contributed equally and significantly in writing this article. All authors read and approved the final manuscript.

\section{Author details}

${ }^{1}$ School of Mathematics and Statistics, Longdong University, Qingyang, P.R. China. ${ }^{2}$ School of Mathematics and Statistics, Northwest Normal University, Lanzhou, P.R. China.

\section{Publisher's Note}

Springer Nature remains neutral with regard to jurisdictional claims in published maps and institutional affiliations.

Received: 29 January 2019 Accepted: 21 May 2019 Published online: 29 May 2019

\section{References}

1. Murray, J.D.: Mathematical Biology I: An Introduction. Springer, New York (2013)

2. Skalski, G.T., Gilliam, J.F.: Functional responses with predator interference: viable alternatives to the Holling type II model. Ecology 82(11), 3083-3092 (2001)

3. Arditi, R., Ginzburg, L.R.: Coupling in predator-prey dynamics: ratio dependence. J. Theor. Biol. 139(3), 311-326 (1989)

4. Arditi, R., Saiah, H.: Empirical evidence of the role of heterogeneity in ratio-dependent consumption. Ecology 73(5), 1544-1551 (1992)

5. Dolman, P.M.: The intensity of interference varies with resource density: evidence from a field study with snow buntings, plectrophenax nivalis. Oecologia 102(4), 511-514 (1995)

6. Jos, C., Ellner, S.P.: Testing for predator dependence in predator-prey dynamics: a non-parametric approach. Proc. Royal Soc., Biol. Sci. 267(1453), 1611-1620 (2000)

7. Beddington, J.R.: Mutual interference between parasites or predators and its effect on searching efficiency. J. Anim. Ecol. 44(1), 331-340 (1975)

8. Deangelis, D.L., Goldstein, R.A., O'Neill, R.V.: A model for tropic interaction. Ecology 56(4), 881-892 (1975)

9. Cantrell, R.S., Cosner, C.: Spatial Ecology Via Reaction-Diffusion Equations, vol. 41, p. xvi. Wiley, Chichester (2003)

10. Cantrell, R.S., Cosner, C.: On the dynamics of predator-prey models with the Beddington-DeAngelis functional response. J. Math. Anal. Appl. 257, 206-222 (2001)

11. Hwang, T.W.: Uniqueness of limit cycles of the predator-prey system with Beddington-DeAngelis functional response. J. Math. Anal. Appl. 290(1), 113-122 (2004)

12. Cantrell, R.S., Cosner, C.: Effects of domain on the persistence of populations in a diffusive food-chain model with Beddington-DeAngelis functional response. Nat. Resour. Model. 14(3), 335-367 (2001)

13. Hwang, T.W.: Global analysis of the predator-prey system with Beddington-DeAngelis functional response. J. Math. Anal. Appl. 281(1), 395-401 (2003)

14. Dimitrov, D., Kojouharov, H.: Complete mathematical analysis of predator-prey models with linear prey growth and Beddington-DeAngelis functional response. Appl. Math. Comput. 162(2), 523-538 (2005)

15. Kratina, P., Vos, M., Bateman, A., Anholt, B.R.: Functional responses modified by predator density. Oecologia 159(2), 425-433 (2009)

16. Stover, J.P., Kendall, B.E., Fox, G.A.: Demographic heterogeneity impacts density-dependent population dynamics. Theor. Ecol. 5, 297-309 (2012)

17. Li, H.Y., Takeuchi, Y.: Dynamics of the density dependent predator-prey system with Beddington-DeAngelis functional response. J. Math. Anal. Appl. 372(2), 644-654 (2011)

18. Ghergu, M., Radulescu, V.: Turing patterns in general reaction-diffusion systems of Brusselator type. Commun. Contemp. Math. 12(4), 661-679 (2010)

19. Ghergu, M., Radulescu, V.: Nonlinear PDEs. Mathematical Models in Biology, Chemistry and Population Genetics. Springer Monographs in Mathematics. Springer, Heidelberg (2012)

20. Fall, M.M.: Periodic patterns for a model involving short-range and long-range interactions. Nonlinear Anal. 175 , 73-107 (2018)

21. Huang, K.G., Cai, Y.L., Rao, F., Fu, S.M., Wang, W.M.: Positive steady states of a density-dependent predator-prey model with diffusion. Discrete Contin. Dyn. Syst., Ser. B 23(8), 3087-3107 (2018)

22. Fan, S.: A new extracting formula and a new distinguishing means on the one variable cubic equation. Natur. Sci. J. Hainan Teach. Coll. 2, 91-98 (1989) (in Chinese)

23. Lou, Y., Ni, W.M.: Diffusion vs cross-diffusion: an elliptic approach. J. Differ. Equ. 154, 157-190 (1999)

24. Pang, P.Y.H., Wang, M.X.: Strategy and stationary pattern in a three-species predator-prey model. J. Differ. Equ. 200, 245-273 (2004)

25. Crandall, M.G., Rabinowitz, P.H.: Bifurcation from simple eigenvalues. J. Funct. Anal. 8, 321-340 (1971)

26. Shi, J.P.: Persistence and bifurcation of degenerate solutions. J. Funct. Anal. 169, 494-531 (1999)

27. Ni, W.M., Tang, M.: Turing patterns in the Lengyel-Epstein system for the CIMA reaction. Trans. Am. Math. Soc. 357, 3953-3969 (2005)

28. Ni, W.M.: Diffusion, cross-diffusion and their spike-layer steady states. Not. Am. Math. Soc. 45, 9-18 (1998)

29. Takagi, I.: Point-condensation for a reaction-diffusion system. J. Differ. Equ. 61, 208-249 (1986) 